\title{
ARTICLE
}

\section{Development of ROV system to explore fuel debris in the Fukushima Daiichi Nuclear Power Plant}

\author{
So Kamada $^{\mathrm{a}^{*}}$, Michio Kato ${ }^{\mathrm{a}}$, Kazuya Nishimura ${ }^{\mathrm{a}}$, Matthew Nancekievill ${ }^{\mathrm{b}}$, Simon Watson ${ }^{\mathrm{b}}$, Barry Lennox ${ }^{\mathrm{b}}$, \\ Ashley Jones ${ }^{c}$, Malcolm J. Joyce ${ }^{\mathrm{c}}$, Keisuke Okumura ${ }^{\mathrm{d}}$ and Jun-ichi Katakura ${ }^{\mathrm{e}}$
}

\begin{abstract}
${ }^{a}$ National Maritime, Port and Aviation Technologies, 6-38-1 Shinkawa, Mitaka-shi, Tokyo, 181-0004, Japan; ${ }^{b}$ The University of Manchester, Oxford Rd,, Manchester, M13 9PL, United Kingdom; ' Department of Engineering, Lancaster Univeristy, Lancaster, LA1 4YR, United Kingdom; ${ }^{d}$ Nagaoka National University of Technology, 1603-1 Kamitomioka-machi, Nagaoka-shi, Niigata-ken, 940-2188, Japan
\end{abstract}

Instruments and Methods were developed to explore fuel debris at the bottom of the Primary Containment Vessel in the Fukushima Daiichi Nuclear Power Plant. A Remotely Operated Vehicle was designed to explore fuel debris and to investigate the distribution and surface profile of fuel debris at the bottom of the primary containment vessel using a sonar and a compact radiation detector. Several tests were carried out using various facilities to determine the capabilities of the sonar and gamma-ray detector to determine the feasibility of the device.

\section{Keywords: $1 F$; PCV; fuel debris; ROV; sonar; radiation detector}

\section{Introduction}

Reducing radiation exposure to workers is a very important issue in promoting the decommissioning of the Fukushima Daiichi Nuclear Power Plant (1F). Radiation emitted from the fuel debris mainly attributes to dose rate distribution in the Primary Containment Vessel (PCV). In order to estimate dose rate in the PCV, it is required to explore fuel debris and investigate those distributions at the bottom of PCV. Optical cameras such as CCDs have low radiation tolerance in the high dose rate environment of the PCV and light rays are attenuated quickly in water. On the other hand, sonar systems utilize acoustic waves, which do not attenuate as quickly in water and may hence be suitable for surveying submerged fuel debris at the bottom of the PCV. Moreover, compact neutron and gamma-ray detectors can also be used to identify fuel debris and other materials in the PCV.

\section{ROV and devices integrated into ROV}

\section{1. $\mathrm{ROV}$}

The Remotely Operated Vehicle (ROV) designed in this study was developed by the University of Manchester to explore fuel debris at the bottom of the PCV and is named the Aqua Vehicle Explorer for In-situ Sensing (AVEXIS). Figure 1 shows a picture of the AVEXIS [1]. The AVEXIS is cylindrical in shape with a diameter of $150 \mathrm{~mm}$ and a length of $347 \mathrm{~mm}$. It has 5

*Corresponding author. Email: kamada-s@nmri.go.jp thrusters on each end-cap that provide movements forwards, backwards, left, right, up, and down as shown in the right of Figure 1. Two $\mathrm{O}$ rings were attached on each end cap to seal the AVEXIS and make it watertight to up to $100 \mathrm{~m}$ depth.
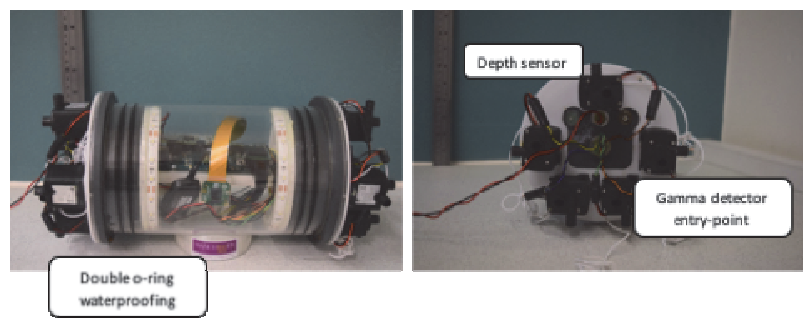

Figure 1. Picture of the AVEXIS, left picture and right picture show front and end of the AVEXIS.

\subsection{Sonar}

The sonar system is able to investigate the distribution and surface profile of fuel debris at the bottom of the PCV in any transparency under the submergible environment in the PCV without being affected by the radiation. Sonar was a rotating-beam sonar 831L [3] with a size of $61 \mathrm{~mm}$ in diameter and 343 $\mathrm{mm}$ in length by IMGENEX Technology Corp., Canada as shown in Figure 2. The frequency of the $831 \mathrm{~L}$ is 2.25 $\mathrm{MHz}$.

\subsection{Compact radiation detector}

The fuel debris can be identified from other objects in the PCV by detecting gamma rays from the fuel debris, 
such as ${ }^{154} \mathrm{Eu},{ }^{60} \mathrm{Co}$, and ${ }^{144} \mathrm{Ce}\left({ }^{144} \mathrm{Pr}\right)$. The $\mathrm{CeBr}_{3}$ inorganic scintillator [2] was used as a photon detector accomdated into the AVEXIS, because the $\mathrm{CeBr}_{3}$ scintillator has higher sensitivity and energy- resolution to gamma rays than those of the well-established $\mathrm{NaI}(\mathrm{Tl})$ scintillator. The $\mathrm{CeBr}_{3}$ scintillator was $10 \mathrm{~mm}$ in diameter and $10 \mathrm{~mm}$ in length and in this study was coupled to a photomultiplier R9880-10 by Hamamatsu Photonics K.K. and covered with an Aluminum housing as shown in Figure 3.

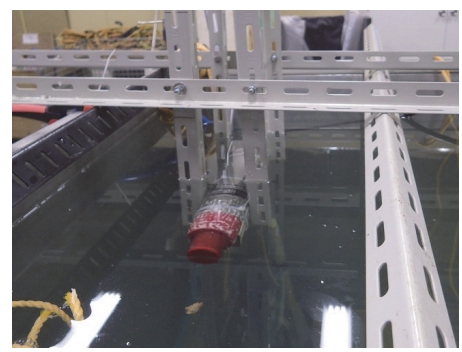

Figure 2. The sonar used in this study was the $831 \mathrm{~L}$ rotatingbeam sonar

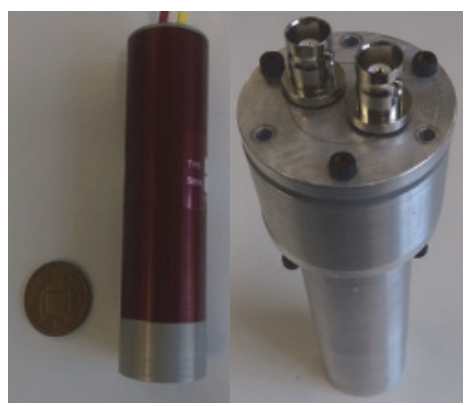

Figure 3. The $\mathrm{CeBr} 3$ inorganic detector without and with aluminum casing.

\section{Tests of sonar system}

\subsection{Tank test}

It is assumed that acoustic noise will be caused by multi-pass propagation of the multiple reflections of the acoustic wave with the wall and the floor of the actual $\mathrm{PCV}$, and this would be increased when the sonar is close to the wall of the PCV. The multiple reflections form "ghost" images behind the first reflection of objects and would be a significant limitation in the exploration of the PCV using sonar.

Two sizes of tank were used to characterize the noise from multi-pass propagation created by the multiple reflections, and produced the mockup PCV with an actual scale of $1 / 6$ to inspect the correlation of the reflections and the shape of the wall and the floor of PCV. Figure 4 shows the mockup of PCV inside a smaller tank. This tank has a dimension of $2 \mathrm{~m} \mathrm{x} 1 \mathrm{~m} \times 1$ $\mathrm{m}$ in depth, and a larger round tank with a of diameter 6 $\mathrm{m}$ and a depth of $10 \mathrm{~m}$ as shown in Figure 5. Moreover, in order to characterize the image resolution of the surface profiling of the fuel debris, two types of test pieces for the fuel debris were provided. One was simulated fuel debris made of copper alloy to identify the debris profile as shown in Figure 6, and the others were stainless steel block with three sizes of roughness, $0 \mathrm{~mm}, 3 \mathrm{~mm}$, and $8 \mathrm{~mm}$ as shown in Figure 7. A rock was also used to confirm the surface status.

In the smaller tank test, the mockup of PCV was also used at the same time, and the model debris was scanned by moving the sonar along the wall.

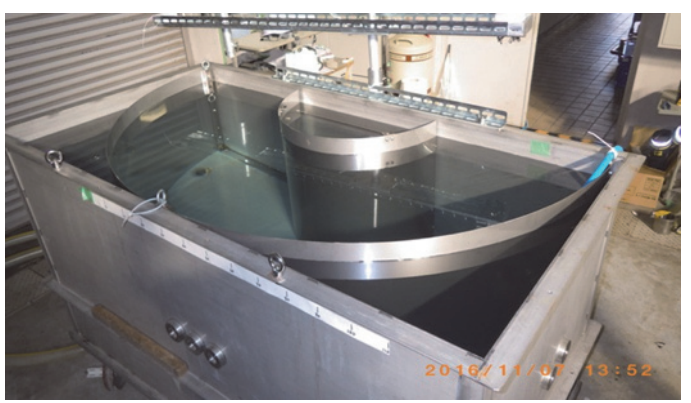

Figure 4. The small tank with the mockup of the PCV.
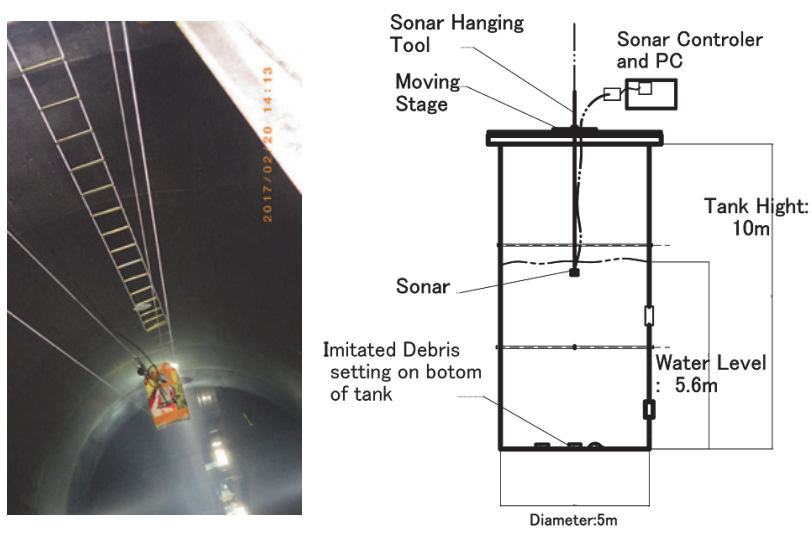

Figure 5. Large round tank.

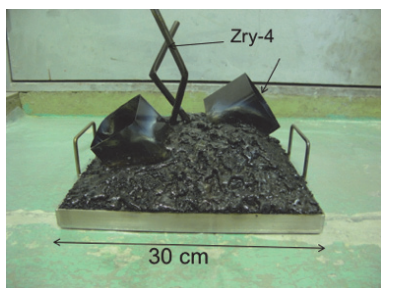

Figure 6. Shape simulated model debris.
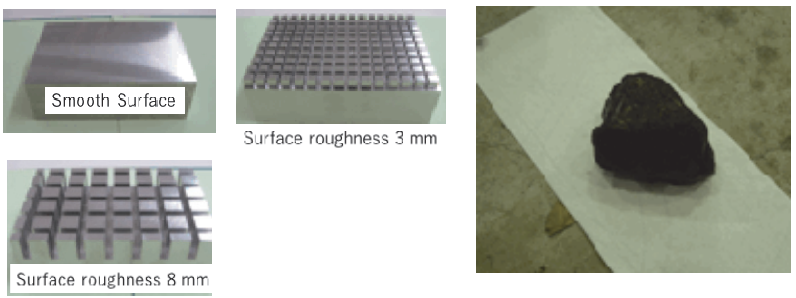

Figure 7. Stainless steel block with roughness and the rock.

\subsection{Irradiation test of compact radiation detector and parts of the $\mathrm{ROV}$}

In order to inspect the radiation tolerance of the compact radiation detector and parts of the ROV, tests were performed at the Dalton Cumbria Facility U.K. that 
can irradiate samples using gamma rays emitted from ${ }^{60} \mathrm{Co}$ with varying dose rate. The samples characterized were the thrusters and control board of the AVEXIS.

\section{Results and discussion}

\subsection{Sonar images}

The $831 \mathrm{~L}$ was able to identify the test pieces of fuel debris in the small tank test as shown in Figure 8, although the ghost due to the reflected wave from water surface was contained in the sonar image. The images by scanning the three stainless steel blocks with a roughness of $8 \mathrm{~mm}$, the rock with a diameter of about 12 $\mathrm{cm}$, and the profile simulated fuel debris are shown in Figures 8,9 and 10. These figures indicate that the $831 \mathrm{~L}$ sonar can distinguish ditches of the stainless steel block as shown in Figures 8, 9. The image by the 831L sonar inside the mock-up of PCV in the small tank shows clearly the profile of the simulated fuel debris in spite of the noises from the multi pass propagations being mixed into the sonar image as shown in Figure 10.

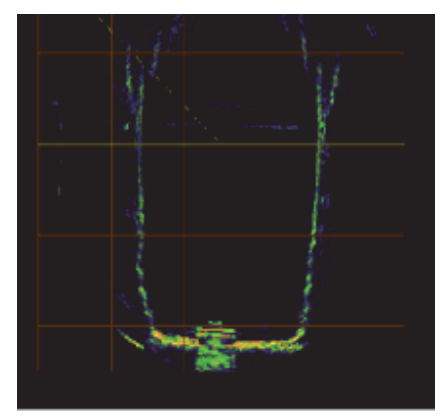

Figure 8. Image of the stainless steel block.
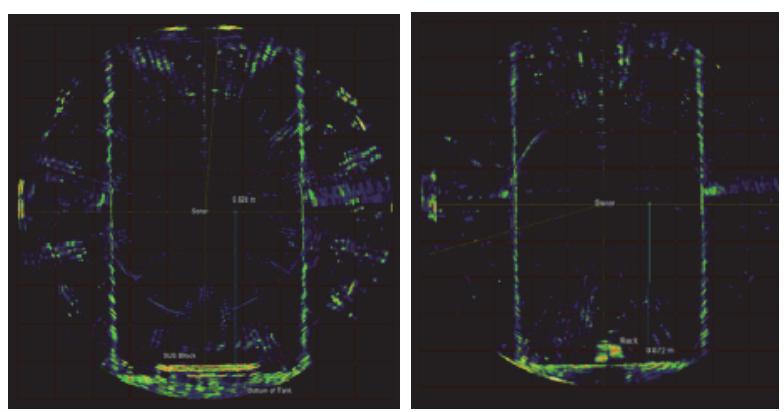

Figure 9. Images of the stainless steel block(left) and the rock(right).

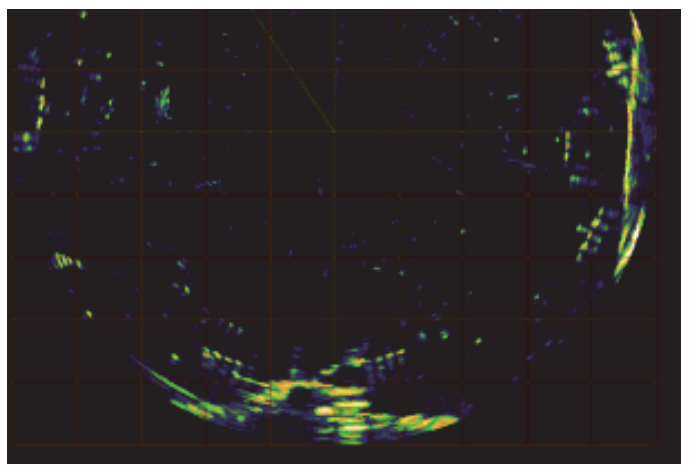

Figure 10. Image of the simulated fuel debris.

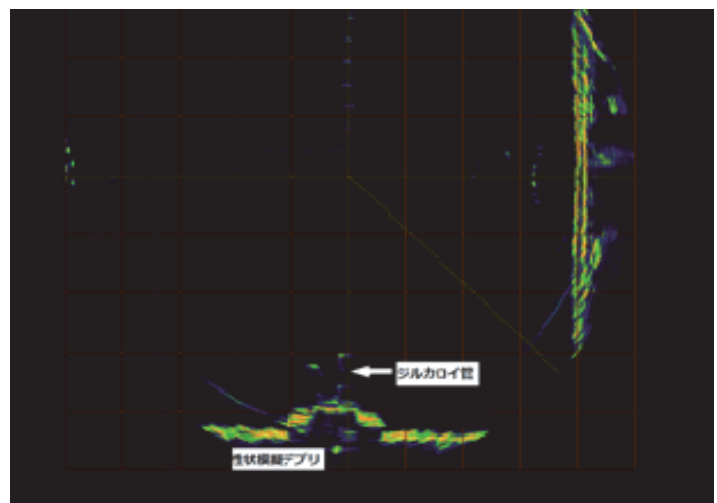

Figure 11. Image of the simulated debris with noise absorber on the wall.

The image by scanning the simulated fuel debris with the $831 \mathrm{~L}$ sonar shows clearly the profile of debris including the Zircaloy pipe as shown in Figure 11.

The test in the large round $(\phi 5 \mathrm{~m})$ tank, for exploring the test-pieces of fuel debris as shown in Figure 12, was carried out by moving the sonar straightforward. The distance between the sonar and the model debris was about $5.5 \mathrm{~m}$. Noise from the multi path propagations was not observed, but the test-pieces of fuel debris could identified as shown in Figure 13.

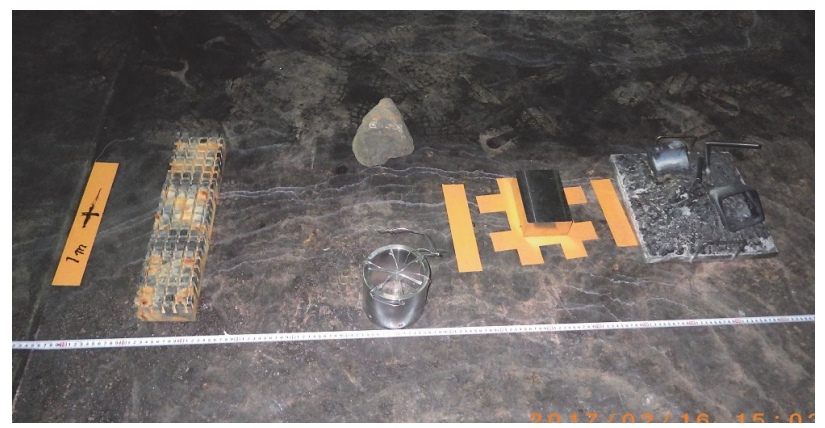

Figure 12. Model debris

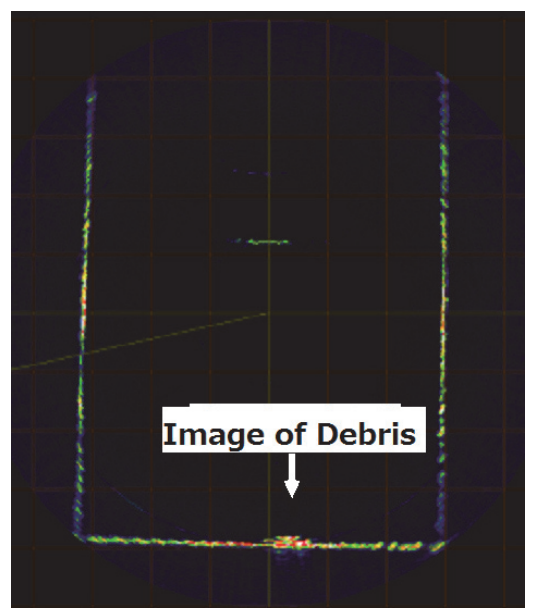

Figure 13. Image of the simulated fuel debris on the bottom of the large tank. 
From the tank test, the $831 \mathrm{~L}$ sonar was preferable to the sonar system accommodated in the AVEXIS. Figure 14 shows a 3D image reconstructed by the 831L sonar data. The simulated debris in the upper picture corresponds to the illustration in the lower picture.

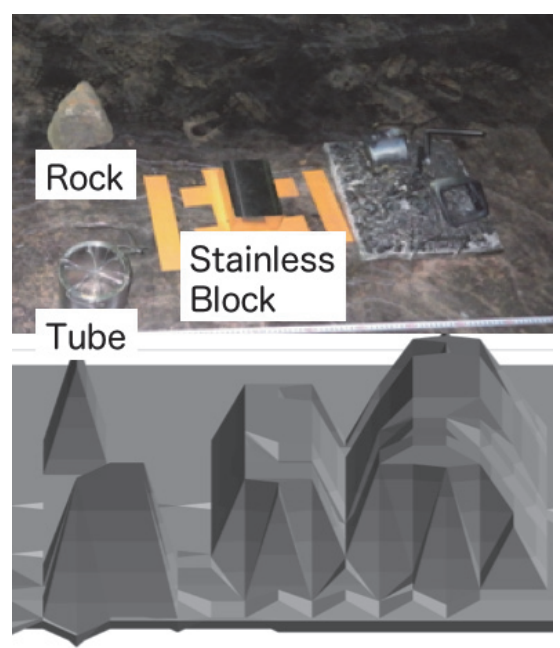

Figure 14. The 3D image reconstructed by the $831 \mathrm{~L}$ sonar data.

\subsection{Irradiation test}

The pumps of the ROV were able to withstand up to 400 Gy of accumulated dose at a dose rate of $91 \mathrm{~Gy} / \mathrm{h}$. The control board of ROV was able to withstand up to 200 Gy of accumulated dose at a dose rate of $44 \mathrm{~Gy} / \mathrm{h}$. The results confirmed that the ROV system could be operated at a dose rate of under $9.9 \mathrm{~Gy} / \mathrm{h}$. Gamma irradiation tests were performed on some components, and indicated that the total ionizing dose (TID) tolerance of the device was greater than $300 \mathrm{~Gy}$ (Si). If assuming a dose rate of about $10 \mathrm{~Gy} / \mathrm{h}$, it was concluded that a target operating life of 10 hours is sufficient

\subsection{Integration test}

The $831 \mathrm{~L}$ sonar was incorporated into the AVEXIS as shown in Figure 15. The communication between the $831 \mathrm{~L}$ sonar and the AVEXIS and the power supply were achieved using a power over Ethernet cable. The operation with the $831 \mathrm{~L}$ sonar in the large tank was no problem.

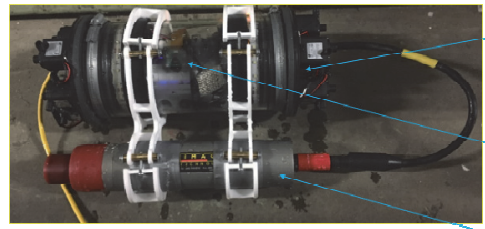

ROV with LED,

HD live camera,

Depth sensor, etc.

Gamma/Neutron

Detector,

Sonar

Figure 15. Integration of the 831L sonar into the AVEXIS.

\section{Summary}

As a result of this investigation, the resolution of shape recognition for the debris surface, high ultrasonic techniques, using equipment such as the $831 \mathrm{~L}$ sonar are effective despite changed in water depth (using the different water tanks). The water depth at $1 F^{\prime}$ s PCV is 6 $\mathrm{m}$ or less. From the result of the irradiation test, the ROV with mounted sonar and radiation sensors, is able to approach fuel debris through detection of gamma rays and neutrons, is able to locate the presence of fuel debris.

The $3 \mathrm{D}$ image could be reconstructed by $831 \mathrm{~L}$ sonar data. In the future, neutron dose from fuel debris may be evaluated by converting from 3D image of sonar output at the time of exploration into geometrical input of a dose evaluation system in PCV.

\section{Acknowledgements}

This work was funded by the Ministry of Education, Culture, Sports, Science and Technology(MEXT) and the Engineering and Physical Sciences Research Council (EPSRC: EP/N017749/1) in the U.K.

\section{References}

[1] A. Griffiths, A. Dikarev, P.R. Green, B. Lennox, X. Poteau and S. Watson, AVEXIS-Aqua vehicle explorer for in-situ sensing, IEEE Robotics and Automation Letters, 1 (2016), pp. 282-287.

[2] W. Drozdowski et al., CeBr3 scintillator development for possible use in space missions, IEEE Trans. Nucl. Sci., 55 (2008), pp.1391-1396.

[3] A.R. Jones, M.J. Joyce, A. Griffiths, B. Lennox, D. Potts, S.A. Watson, J. Katakura, K. Okumura, K. Kim, M. Katoh, K. Nishimura and K. Sawada, Radiation hardness testing of an organic liquid scintillation detector for use in high dose rate accident response scenarios, Proceedings of HOTLAB 2016, Karlsruhe Germany, Oct. 2-6, 2016, (2016), pp.1-4. 